\title{
MARK2 inhibits the growth of HeLa cells through AMPK and reverses epithelial-mesenchymal transition
}

\author{
GE XU ${ }^{1}$, YINGGANG GE ${ }^{2}$, XIAOHONG TAO ${ }^{3}$, QING GAO $^{3}$ and XIAOYAN LIANG ${ }^{3}$ \\ ${ }^{1}$ Institute of Life Science, Chongqing Medical University; Departments of ${ }^{2}$ Gastrointestinal Surgery and \\ ${ }^{3}$ Gastroenterology, The First Affiliated Hospital of Chongqing Medical University, Chongqing 400016, P.R. China
}

Received February 18, 2017; Accepted May 3, 2017

DOI: 10.3892/or.2017.5686

\begin{abstract}
Microtubule affinity-regulating kinases (MARKs; MARK1, MARK2, MARK3 and MARK4) act directly downstream of LKB1, the multitasking tumor-suppressor kinase, and thereby mediate its biological effects. Current understanding of the function of MARKs is greatly restricted to regulation of cell polarity. However, whether or how MARKs contribute to cellular growth control remains largely unknown. In the present study, we utilized an inducible lentiviral expression system that allows rapid MARK expression in LKB1-deficient HeLa cells, and characterized additional functions of MARKs: overexpression of MARK2 in HeLa cells resulted in a decrease in cell growth, inhibition of colony formation and arrest in G1 cell cycle phase, with AMPK as the putative downstream effector upregulating the expression of p21 and p16. MARK2 was found to play a role in F-actin reorganization and to contribute to reversal of epithelial-mesenchymal transition (EMT) as exemplified in the case of HeLa cells that exhibited phenotypic changes, reduced cell migration and invasion. Our findings unveil the coordinated regulation of cell growth and EMT mediated by MARK2, and also provide new insights into the mechanisms underlying the anti-metastatic activity of MARK2.
\end{abstract}

\section{Introduction}

The tumor-suppressor LKB1 is an evolutionarily conserved and ubiquitously expressed serine/threonine kinase, which can be inactivated either by germline mutations resulting in

Correspondence to: Dr Xiaoyan Liang, Department of Gastroenterology, The First Affiliated Hospital of Chongqing Medical University, 1 You Yi Road, Chongqing 400016 P.R. China

E-mail: 1xyan925@hotmail.com

Abbreviations: AMPK, adenosine monophosphate-activated protein kinase; MARK, microtubule affinity-regulating kinase; CDK, cyclindependent kinase; EMT, epithelial-mesenchymal transition

Key words: MARK, AMPK, EMT, LKB1, HeLa cell line
Peutz-Jeghers syndrome or by somatic mutations causing predisposition to multiple sporadic cancers (1-3). Termed a 'master kinase' LKB1 directly phosphorylates and activates a set of 14 kinases from the adenosine monophosphate (AMP)-activated protein kinase (AMPK) family, which includes AMPK (AMPK- $\alpha 1$ and AMPK- $\alpha 2)$, brain-specific kinases (BRSKs; BRSK1 and BRSK2), microtubule affinity-regulating kinases (MARKs; MARK1, MARK2, MARK3 and MARK4), salt-inducible kinases (SIKs; SIK1, SIK2 and SIK3), nua (novel)/SNF1-like kinases (NUAK1 and NUAK2), and the Snf-related serine/threonine kinase (SNRK) $(4,5)$. Through these downstream kinases, LKB1 regulates multiple cellular processes contributing to tumor suppression, including cell cycle regulation, cell polarity establishment, energy metabolic balance and apoptosis control (6-8). However, the underlying molecular mechanisms are still not completely understood.

The majority of the known functions of LKB1 kinase are mediated by its ability to activate AMPK, a central conserved regulator of energy metabolism and cell growth (9). When cellular AMP:ATP ratios rise, LKB1 directly phosphorylates the threonine 172 (Thr172) in the activation loop of AMPK and results in an increase in ATP-producing activities and a decrease in ATP-consuming processes including essentially all biosynthetic pathways required for cell growth $(10,11)$. Previous investigations have shown that activation of the LKB1-AMPK pathway is able to suppress cell growth by arresting the cell cycle in the $\mathrm{G} 1$ phase $(12,13)$. In a similar manner, we confirmed and extended these observations by demonstrating that exogenous activation of LKB1-AMPK signaling induces downregulation of cyclins (cyclin D1 and D3) and upregulation of the cyclin-dependent kinase (CDK) inhibitors (p53, p21 and p16), and thus, inhibits G1/S cell cycle transition, even in cells with endogenous expression of LKB1 (14).

Disruption of polarity, as uncontrolled cell growth, is a quintessential characteristic of epithelial-derived cancer cells, and is critical in epithelial-mesenchymal transition (EMT), a crucial step during tumor invasiveness, metastasis and fibrosis $(15,16)$. LKB1 has been firmly established as a highly conserved regulator of cell polarization, and there is growing evidence that MARKs may be primary mediators of LKB1 action in that process $(17,18)$. In epithelial cells, the polarizing activity of LKB1 kinase is relayed through MARKs, which phosphorylate microtubule-associated 
proteins including tau, and increase the dynamic instability of microtubules $(19,20)$. According to current knowledge, LKB1 signaling predominantly regulates cell growth via AMPK and cell polarity via MARKs. However, Müller et al suggested that MARK2 also plays a role in the regulation of cell proliferation by phosphorylating the cell cycle regulatory phosphatase Cdc25 resulting in a complex of Cdc25 and 14-3-3 and cell cycle blockage (21), which indicates that LKB1-mediated regulation of cell growth and polarity signaling are not separate, but connected to each other. However, how these two pathways are orchestrated to maintain cellular homeostasis has not been fully investigated. To address this question and to better understand MARK signaling, we enforced MARK expression using a lentiviral system in adherent HeLa cells, and revealed a dual role of MARK2 in the regulation of AMPK-mediated G1/S transition and the actin-based cytoskeletal system, indicating that cell proliferation and cell polarity may be broadly integrated under the control of MARK2 signaling.

\section{Materials and methods}

Materials. Cell culture media and supplements were purchased from Invitrogen Life Technologies (Carlsbad, CA, USA). Antibodies targeting MARK1, MARK2, MARK3, MARK4, AMP-activated protein kinase $\alpha$ (AMPK $\alpha)$, phospho-AMPK $\alpha$ (Thr172), p21, E-cadherin, N-cadherin and vimentin were purchased from Cell Signaling Technology (Beverly, MA, USA). Antibodies against p53, p16 and glyceraldehyde-3-phosphate dehydrogenase (GAPDH) were obtained from Santa Cruz Biotechnology (Santa Cruz, CA, USA). Horseradish peroxidase (HRP)-conjugated secondary antibodies were purchased from Jackson ImmunoResearch Laboratories (West Grove, PA, USA). Phalloidin-tetramethylrhodamine-conjugated (phalloidin-TRITC) and compound C were purchased from Sigma-Aldrich (St. Louis, MO, USA). Unless otherwise noted, chemicals and organic solvents were obtained from Sigma-Aldrich and were of the highest grade.

Cell culture and viral infection. Human cervical cancer cell line HeLa cells were purchased from the Type Culture Collection of the Chinese Academy of Sciences (Shanghai). Cells were maintained as frozen stocks. After viral infections, only pooled clones were used to avoid clonal variations. Culturing of cells was performed as previously described (22).

Lentivirus production and transduction. Expression plasmids of MARKs were obtained from Professor Dario Alessi (University of Dundee). LV5-GFP-Puro lentiviral vectors expressing MARKs (LV-MARK1, LV-MARK2, LV-MARK3 and LV-MARK4) were constructed by GenePharma Co., Ltd. (Shanghai, China). HeLa cells were infected with LV-MARKs or LV-GFP mock vector at multiplicities of infection (MOI) of 20 for $48 \mathrm{~h}$ in the presence of $8 \mu \mathrm{g} / \mathrm{ml}$ Polybrene (Sigma, St. Louis, MO, USA) and maintained in puromycin.

Cell proliferation assay. Cell proliferation was examined using a 3-(4,5-dimethylthiazol-2-yl)-5-(3-carboxymethoxyphenyl)2-(4-sulfonphenyl)-2H-tetrazolium (MTS) assay (CellTiter 96 AQueous One Solution Cell Proliferation Assay; Promega, Madison, WI, USA), according to the manufacturer's instruc- tions. Cells (1,000/well) were seeded onto 96-well plates in triplicates and incubated in culture medium containing MTS every day for 4 days. Absorbance was measured at $490 \mathrm{~nm}$ in a SpectraMax M5 (Molecular Devices, Sunnyvale, CA, USA). Experiments were repeated 3 times and representative results are shown.

Soft agar colony formation assay. Cells were seeded at a density of $1 \times 10^{3}$ cells in 60-mm dishes in complete media with a bottom layer of $0.6 \%$ low melting point (LMP) agar and a top layer of $0.35 \%$ low LMP agar. The plates were incubated at $37^{\circ} \mathrm{C}$ in a humidified incubator for 10-14 days. Cells were fed twice a week by adding fresh medium. Colonies were stained with crystal violet, photographed and manually counted.

Flow cytometric analysis. Exponentially growing cells were infected with the indicated lentiviral vectors for $48 \mathrm{~h}$, detached and fixed with $70 \%$ ethanol overnight. Propidium iodide/RNase A was used to stain the nuclei. Cell cycle distribution was determined by flow cytometry using a FACSVantage SE cell sorter (BD Biosciences, Franklin Lakes, NJ, USA). Percentage of cells at different phases of the cell cycle was analyzed using the CellQuest ${ }^{\mathrm{TM}}$ Pro software (BD Biosciences).

Western blot analysis. Total proteins were analyzed and blotted as previously described $(22,23)$. Compound $\mathrm{C}(\mathrm{CC} ; 20 \mu \mathrm{M})$ or dimethyl sulfoxide (DMSO) was added $24 \mathrm{~h}$ post infection. Specific primary antibodies recognizing MARK1 $(1: 1,000)$, MARK2 (1:1,000), MARK3 (1:1,000), MARK4 (1:1,000), AMPK $\alpha(1: 1,000)$, phospho-AMPK $\alpha$ (Thr172) $(1: 1,000)$, p53 (1:1,000), p21 (1:500), p16 (1:500), E-cadherin (1:1,000), $\mathrm{N}$-cadherin $(1: 1,000)$, vimentin $(1: 1,000)$ and GAPDH $(1: 1,000)$ were used at the indicated dilutions. HRP-conjugated secondary antibodies were used at 1:10,000 dilutions. Signals were detected by Substrate SuperSignal West Pico Chemiluminescent Substrate kit (Pierce Biotechnology, Rockford, IL, USA), and quantified using Quantity One software (Bio-Rad, Hercules, CA, USA).

Cell staining and confocal imaging. Cells grown on glass coverslips were fixed with $4 \%$ paraformaldehyde and permeabilized in $0.1 \%$ Triton $\mathrm{X}-100$. F-actin was visualized using TRITC-conjugated phalloidin (phalloidin-TRITC). Nuclei were counterstained with 4,6-diamidino-2-phenylindole (DAPI). Digital images of 10 random fields ( $>500$ cells) were used to calculate the percentage of elongated cells. Cells were classified as elongated when the length of the protrusion was 2-fold longer than the width of the cell body (24). Representative images were captured using an inverted confocal microscope (FluoView FV1000; Olympus, Tokyo, Japan) at a magnification of $\mathrm{x} 800$.

Wound-healing assay. HeLa cells were seeded into 6-well plates and infected with MARK2 or green fluorescence protein (GFP) mock lentiviruses. Confluent cell monolayers were scratched with micropipette tips, and images were captured at each time courses for $48 \mathrm{~h}$. The wound healing capacities were calculated by measuring the distance of the migrating edge. Mean values were obtained from 3 separate experiments. 

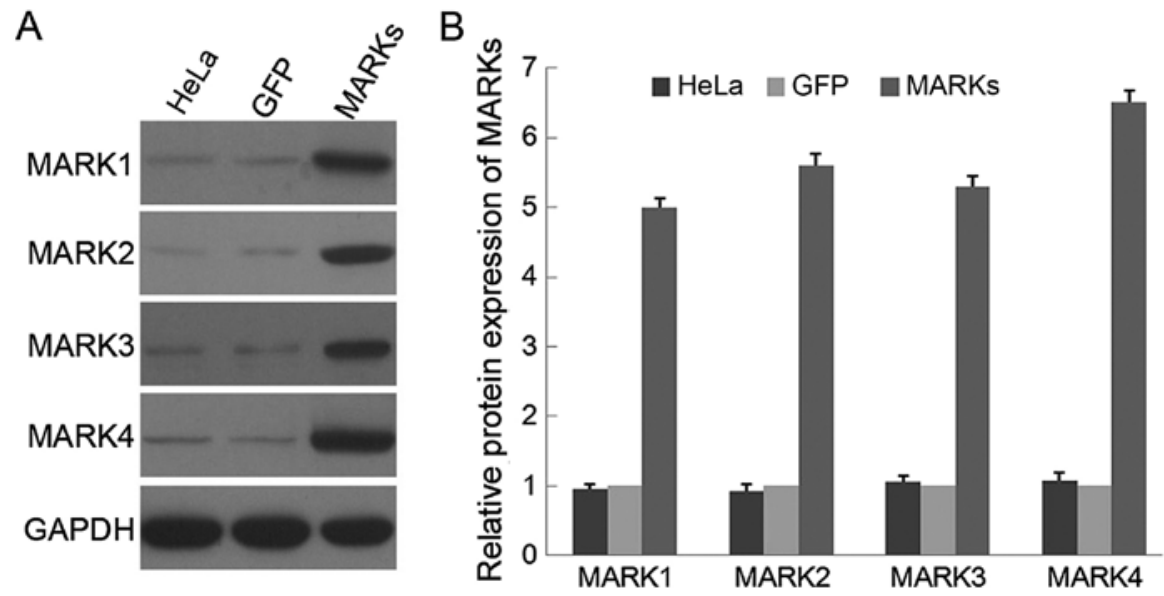

Figure 1. Lentiviral-mediated overexpression of MARKs in the HeLa cells. HeLa cells were transduced with lentiviral expression constructs of MARKs or a GFP mock vector for $48 \mathrm{~h}$. (A) Protein expression profile was determined using western blot analysis. GAPDH was used as a loading control. (B) The relative expression of the protein levels of MARKs are presented as compared to the GFP control. Values represent 3 independent experiments.

Transwell invasion assay. Cell invasion assays were performed using Transwell chambers with $8-\mu \mathrm{m}$ pores (Corning Costar Corp., Cambridge, MA, USA) according to the manufacturer's instructions. Infected cells were seeded on top of the Matrigel in the upper chamber, and the bottom chamber was filled with culture medium containing $10 \%$ fetal bovine serum (FBS), as the chemoattractant for $24 \mathrm{~h}$. The invasive cells on the underside of the filter, were fixed with paraformaldehyde, stained with crystal violet and counted. Experiments were performed in triplicate.

Statistical analysis. Data are presented as mean \pm standard deviation (SD). Unless otherwise indicated, cell culture experiments were reproduced 3 or more times. Paired t-tests were used to determine statistical significance. p-values $<0.05$ were considered statistically significant.

\section{Results}

Lentiviral transduction of MARKs into HeLa cells. MARKs have been identified as important components of LKB1-related signal transduction pathways in a variety of species $(4,5)$. In the present study, LKB1-deficient HeLa cells were transduced with lentiviral vectors expressing 4 isoforms of MARKs respectively. Western blot analysis confirmed a 5- to 6-fold increase in protein expression of 4 MARK kinases in the HeLa cells infected with the corresponding MARK lentivirus compared with that in cells infected with a GFP mock vector (Fig. 1). Uninfected HeLa cells were also included, and no difference was observed in the expression of MARK protein between the parental HeLa and HeLa cells infected with the GFP vector. Thus, an efficient overexpression of MARKs was successfully achieved in the LKB1-null HeLa cells.

Overexpression of MARK2 inhibits proliferation of $\mathrm{HeLa}$ cells. It is known that reintroducing LKB1 into HeLa cells restores LKB1 activity and induces growth suppression $(25,26)$. As important downstream kinases regulated by LKB1, MARKs may also be involved in growth inhibition. To investigate this possibility, we performed MTS assay, which

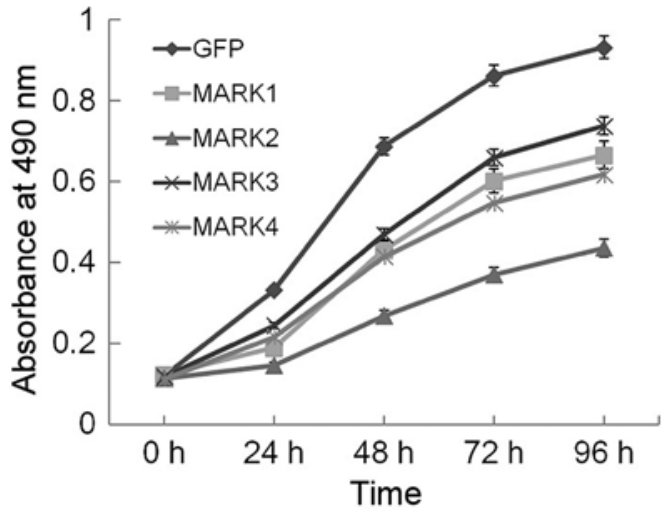

Figure 2. MARK2 overexpression inhibits the proliferation of HeLa cells HeLa cells were infected with a lentivirus expressing 4 different isoforms of MARKs or a GFP empty vector. An MTS assay was used to measure cell number at the indicated time points. Experiments were repeated 3 times and representative results are shown.

is used as colorimetric method for sensitive quantification of viable cells in proliferation (27). Over the 96-h interval examined (Fig. 2), growth curve experiments clearly demonstrated that enforced expression of MARKs conferred a proliferative disadvantage to HeLa cells, which was most evident in the MARK2-transduced cells and suggests that overexpression of MARKs, particularly MARK2, strongly inhibited the proliferation of HeLa cells.

Overexpression of MARK2 inhibits anchorage-independent growth of HeLa cells. Anchorage-independent growth of cells in soft agar is one of the hallmark characteristics of cellular transformation and uncontrolled cell growth (28). The LKB1-deficient HeLa cell line is one widely used human cervical cancer line, and forced LKB1 expression reverses the oncogenesis of HeLa cells. To explore whether enhanced expression of MARKs had a similar effect, we performed a soft agar colony formation assay. Notably, the HeLa cells infected with the GFP control vector grew efficiently in soft agar and formed numerous colonies, whereas the MARK2-overexpressing cells exhibited a 

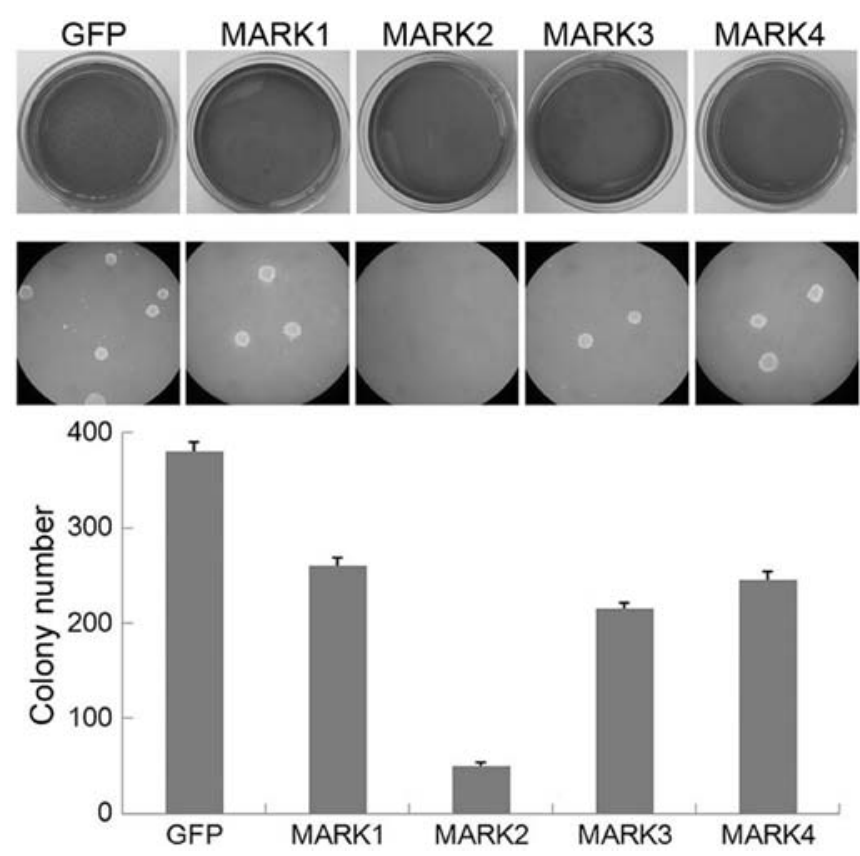

Figure 3. MARK2 overexpression inhibits anchorage-independent growth of HeLa cells. HeLa cells were infected with a lentivirus expressing 4 different isoforms of MARKs or a GFP empty vector. Anchorage-independent growth in soft agar was observed in the indicated cells. Experiments were repeated 3 times and representative results are shown.
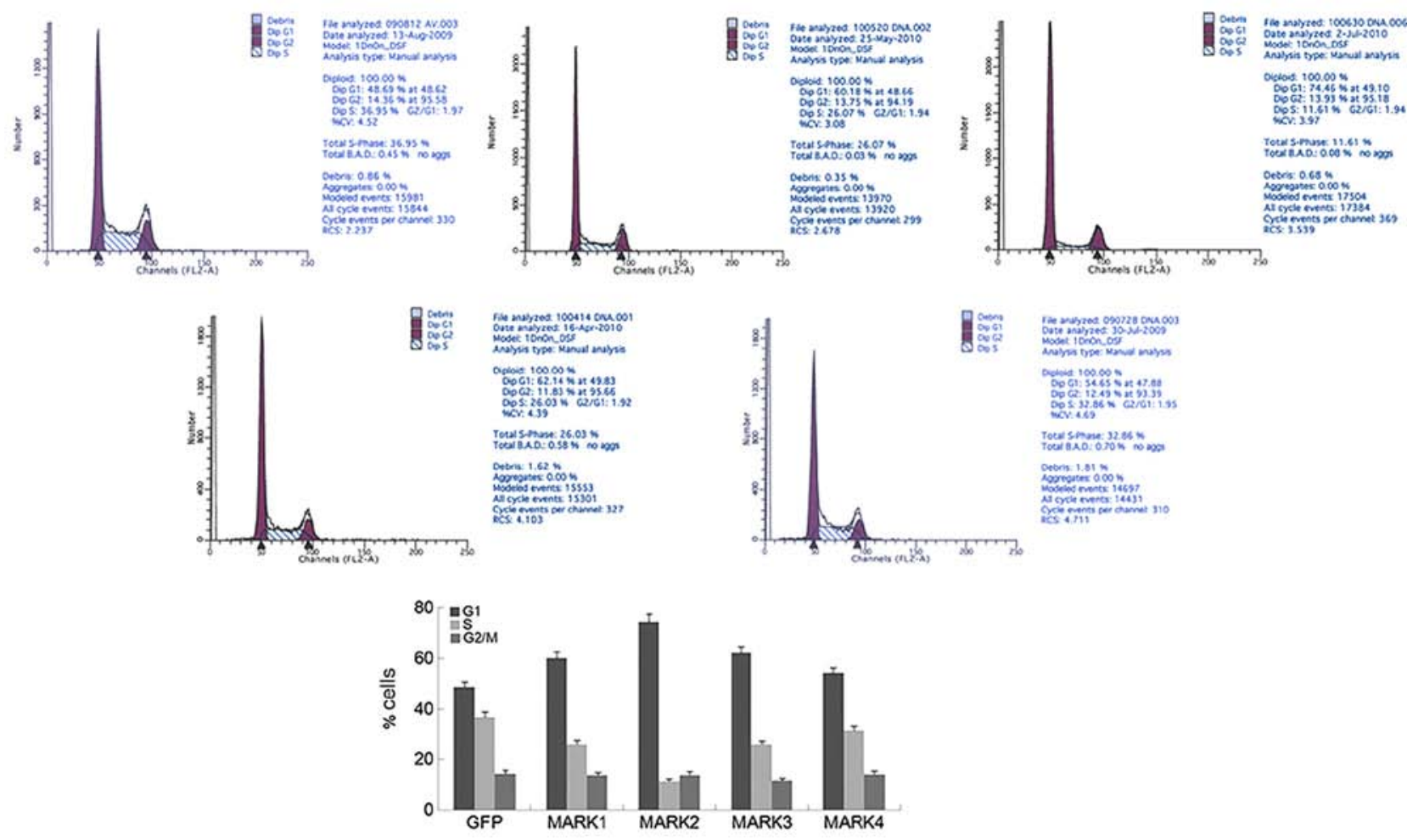

Figure 4. MARK2 overexpression increases the population of cells in the $\mathrm{S}$ phase of the cell cycle. HeLa cells were infected with MARK lentivirus for $48 \mathrm{~h}$, and were then analyzed by flow cytometry. Cell cycle profile and phase distribution are presented. Data are representative of 3 independent experiments.

significant reduction in anchorage-independent growth on soft agar (Fig. 3). Overexpression of MARK1, MARK3 or MARK4 led to minor attenuation of colony formation. These results suggest that overexpression of MARK2 inhibits the anchorage-independent growth of HeLa cells, which mimics the effect of the upstream kinase LKB1.
Overexpression of MARK2 arrests the cell cycle at G1 phase. In mammalian cells, proliferation control is primarily achieved in the G1 phase of the cell cycle (29). Previous studies have provided compelling evidence that ectopic LKB1 expression in LKB1-deficient cancer cells inhibits G1/S transition and arrests cells in the G1 phase $(25,30,31)$. Transduction of MARK2 into 


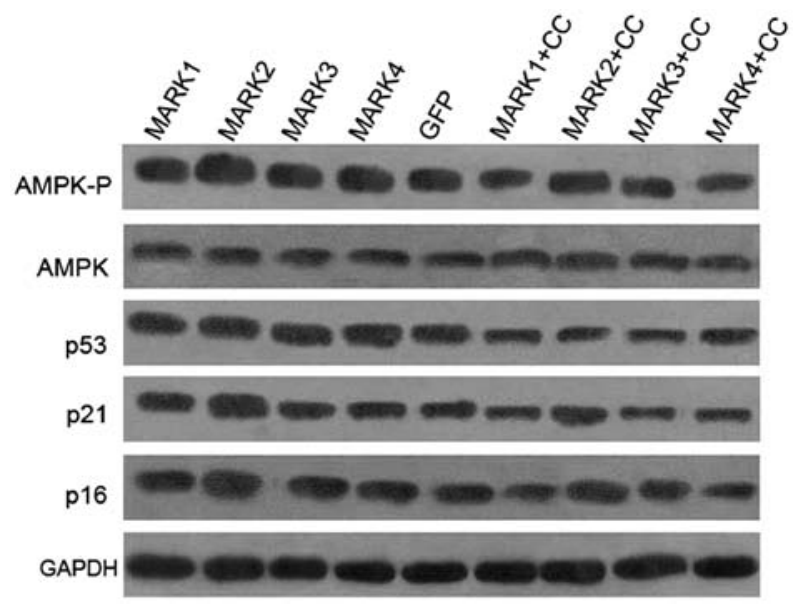

Figure 5. MARK2 overexpression increases AMPK $\alpha$ phosphorylation and the protein levels of p21 and p16, but not p53. HeLa cells infected with indicated lentiviral vectors for $48 \mathrm{~h}$. Expression of total AMPK $\alpha$, phosphorylated AMPK $\alpha$ (Thr172), p53, p21 and p16 was assessed using western blot analysis. GAPDH was used as a loading control.

HeLa cells led to increased accumulation of cells in G1 and a reduced proportion of cells in $\mathrm{S}$ phase, compared with that in cells infected with the GFP vector (Fig. 4). Similar but less pronounced changes in cell cycle profile were observed for the other 3 MARKs. These results indicate that exogenous expression of MARK2 is sufficient to induce G1 arrest in cells with endogenous LKB1 expression deficiency.

Overexpression of MARK2 activates AMPK and increases expression of $p 21$ and p16, but not p53. The role of AMPK as a key mediator in LKB1-related signaling cascades raises the possibility that it may play a role in the observed intracellular cell cycle modulation by MARK2. Overexpression of MARK2 promoted phosphorylation of AMPKa at Thr172 in the HeLa cells, while the protein level of AMPK $\alpha$ was unaffected (Fig. 5), suggesting that inducible expression of MARK2 activated AMPK in the HeLa cells. No observable differences were detected in AMPK phosphorylation in the HeLa cells transduced with MARK1, MARK3 or MARK4 lentiviral vectors.

Induction of CDK inhibitors is a well-characterized mechanism through which to inhibit G1/S transition (32). To investigate whether MARKs mediate cell cycle arrest by regulating expression of CDK inhibitors, we analyzed the expression levels of p21, p16 and p53 protein in the HeLa cells. The results presented in Fig. 5 indicate a specific increase in p21 and p16 levels in the MARK2-transduced cells, whereas the levels of p53 were not changed. Furthermore, to test whether AMPK mediates the cell cycle-controlling functions of MARK2, we used compound $\mathrm{C}$, a potent and selective inhibitor of AMPK. As expected, treatment with compound $\mathrm{C}(\mathrm{CC})$ was able to repress AMPK $\alpha$ phosphorylation in all groups (Fig. 5), further confirming that compound $\mathrm{C}$ is an effective inhibitor of AMPK activity. Induction of $\mathrm{p} 21$ and $\mathrm{p} 16$ expression in MARK2 transduced cells was markedly suppressed by compound C (Fig. 5), suggesting that in HeLa cells AMPK is a critical downstream effector of MARK2, and AMPK kinase activity is required for p21 and p16 induction.
No differences were observed in the expression of p21, p16 or p53 protein after transduction with MARK1, MARK3 or MARK4 lentivirus. Additionally, inactivation of AMPK using pharmacologic inhibitor, compound $\mathrm{C}$, attenuated the expression of p53, p21 and p16 in all groups, which is in good agreement with our previous study that activation of AMPK signaling inhibits $\mathrm{G1} / \mathrm{S}$ progression by upregulating the $\mathrm{p} 53$, p21 and p16 pathways (14).

Overexpression of MARK2 reverses the EMT phenotype of HeLa cells. MARKs are thought to function as regulators of cell polarity since their activation is associated with cytoskeletal modification $(19,20)$. Next, we examined whether overexpression of MARKs was able to reorganize the actin cytoskeleton and restore HeLa cells to an epithelial phenotype. Analysis of the actin cytoskeleton was performed by fluorescence microscopy following phalloidin staining. As shown in Fig. 6A, parental HeLa and HeLa cells infected with the GFP vector display elongated and spindle-shaped phenotypic characteristics of mesenchymal cells, with transcellular filamentous actin (F-actin) stress fiber formation, whereas overexpression of MARK2 induced the typical cobblestone-like epithelial characteristic phenotype, with cortical actin staining and actin filament bundles below the plasma membrane. MARK2 overexpression significantly decreased the elongated (mesenchymal-like) cell population of HeLa cells in comparison to the GFP control vector (73 vs. 30\%) (Fig. 6B). Additionally, overexpression of MARK1, MARK3 or MARK4 induced a morphological switch to create an epithelial cell population, in a similar, but less profound way. Western blot analysis further showed upregulation of the epithelial marker E-cadherin, and downregulation of the mesenchymal marker $\mathrm{N}$-cadherin and vimentin in MARK2-overexpressing HeLa cells (Fig. 6C). These results indicate that enforced expression of MARK2 may remodel the actin cytoskeleton and inhibit the development of a mesenchymal-like phenotype of HeLa cells, uncovering the MARK2-negative role in regulating the EMT process.

Overexpression of MARK2 suppresses cell migration and invasion of HeLa cells. The finding that MARK2 appears to influence EMT prompted us to more closely examine its effects on cell migration and invasion. To assay collective cell migration, we performed scratch wound assay of cell monolayers. As expected, we observed delayed closure upon overexpression of MARK2 compared with the control cells (Fig. 6D). Statistical analysis indicated that the migratory activity of control HeLa cells was $\sim 3$-fold higher than that of the MARK2-infected cells (Fig. 6E). We also used Matrigelcoated Transwell chambers to assess cell invasiveness and yielded similar results (Fig. 6F). The invasive capacity of the MARK2-transduced cells was decreased to $\sim 10 \%$ of the control cells (Fig. 6G). These results indicate that enhanced expression of MARK2 led to inhibited cell migration and invasion, which is a characteristic feature of EMT.

\section{Discussion}

Among the numerous molecular mechanisms altered in human cancers, those involving cell division cycle control are believed fundamental for oncogenesis (33). Originally discovered as 

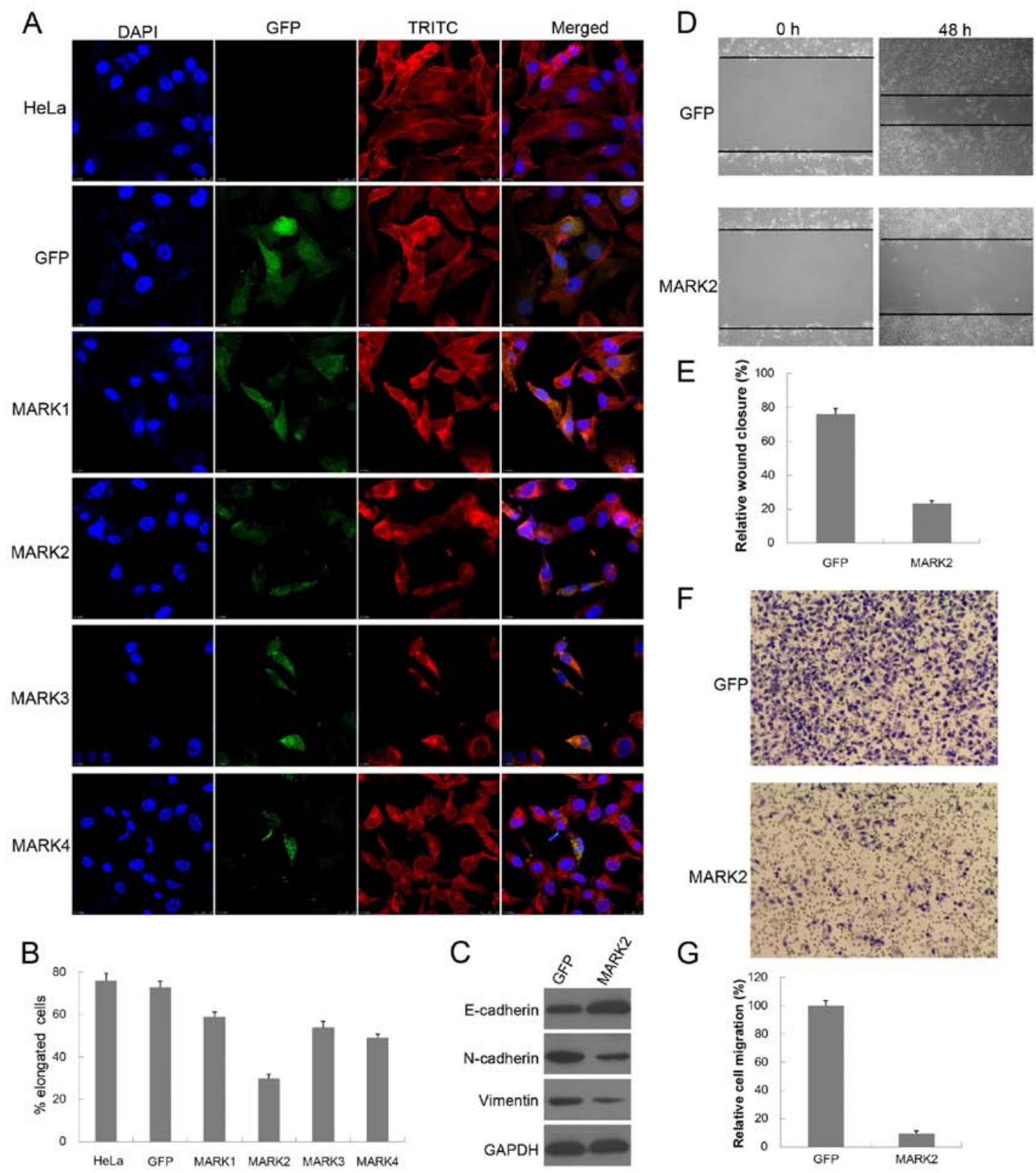

G

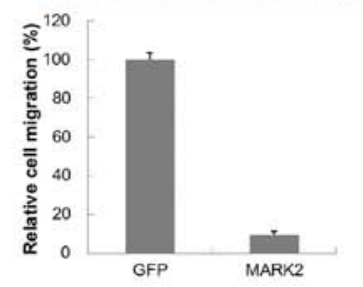

Figure 6. MARK2 overexpression inhibits EMT phenotypes and reduces cell migration and invasion. HeLa cells were infected with indicated lentiviral vectors for $48 \mathrm{~h}$. (A) Cells were analyzed for morphological changes by confocal microscopy, and visualized with DAPI (blue), phalloidin-TRITC (red) and EGFP (green). (B) The ratios of elongated cells were calculated from the number of elongated cells and the total number of adherent cells by microscopic observation. Parental HeLa cells were also included. (C) Western blot analysis of E-cadherin, N-cadherin and vimentin was performed. GAPDH served as a loading control. (D) Wound healing assays were performed. Representative images were captured at 0 and $48 \mathrm{~h}$ after wounding with a magnification of $\mathrm{x} 100$. (E) Quantification was carried out by measuring the distance migrated compared with the controls. (F) Transwell migration assays were performed with HeLa cells infected with MARK2 lentivirus or GFP vector for 24 h. Representative images were captured with a magnification of x100. (G) Quantification was carried by counting the number of migratory cells that had infiltrated the filter.

polarity proteins, the importance of MARKs has been greatly limited to the regulation of cell polarization in a contextdependent manner. However, by utilizing lentiviral systems in LKB1-deficient HeLa cells, we revealed that overexpression of MARK2 led to retarded cell growth, decreased colony formation and cell cycle arrest, which suggests that MARK2 may be an important regulator of cell proliferation and provides further insights into the roles of MARK2 in anti-tumorigenesis. The suppressive mechanism of HeLa cell proliferation by MARK2 appeared to be cell cycle arrest, rather than apoptosis, since the cell population of the G1 phase, but not G0 phase was significantly increased by MARK2 overexpression. In addition, the
HeLa cell line is severely impaired in LKB1 activity (30). The present study showed that inducible expression of MARK2 in HeLa cells was sufficient to mimic LKB1 signaling activation as previously reported (12-14), and further confirms that MARK2 is a critical downstream target of LKB1.

MARKs and AMPK belong to the same protein kinase family, referred as AMPK-related kinase family, and are well-established substrates of LKB1 signaling $(4,5)$. In the present study, we found that enforced expression of MARK2 induced phosphorylation of AMPK, and led to the upregulation of p21 and p16 in an AMPK-dependent manner. The present study demonstrated that MARK2 upregulation could 
be responsible for the AMPK-mediated increase of p21 and p16 protein expression; this increased expression is of critical importance in the induction of G1 arrest and cell growth inhibition, as widely reported in the literature $(34,35)$. To the best of our knowledge, this is the first study to report that AMPK is a novel and essential downstream target of MARK2 signaling and acts as an important factor during MARK2-induced G1 arrest, although additional analyses are required to precisely define how MARK2 induces AMPK activation. Our findings showcase the complexity of MARK2 biology and indicate that cell growth and polarity pathways mediated by LKB1 are intimately connected to each other.

Induction of CDK inhibitors is a well-characterized mechanism to inhibit the activity of cyclin/CDK complexes and prevent cell cycle progression (32). Notably, in the present study, overexpression of MARK2 activates AMPK and induces the expression of p21 and p16, but not p53. These results suggest that MARK2-AMPK activation triggers cell cycle blockage in a p53-independent mode, which is not in complete agreement with our earlier study that LKB1-AMPK mediates G1 cell cycle arrest by inducing the expression of p21, p16 and p53 (14). One plausible explanation is that HeLa cells constitutively express human papilloma virus E6 protein, which targets p53 for proteasome-mediated degradation and abrogates its tumor-suppressor function $(36,37)$. This idea is sustained by a previous study which showed that LKB1 forms a complex with transcription regulatory factors LMO4, GATA-6 and Ldb1, and induces GATA-mediated p21 expression and G1 cell cycle arrest through p53-independent mechanism in HeLa cells (38). Further studies are warranted to validate the GATA-related model in the MARK2-AMPK pathway.

In this context, we stained F-actin cytoskeleton with phalloidin and observed that overexpression of MARK2 in HeLa cells led to rearrangement of actin cytoskeleton and formation of stress fibers, supporting a role of MARK2 in the regulation of the actin cytoskeleton in HeLa cells and raising the possibility that MARK2 may interact with the Rho family of small GTPases, which are regarded as central regulators of the actin cytoskeletal system $(39,40)$. Further investigations may elucidate these issues more clearly. Loss of cell polarity, along with the acquisition of motility and invasiveness, are regarded as typical phenomena during the epithelial-mesenchymal transition (EMT) process $(41,42)$. Enforced expression of MARK2 inhibited the mesenchymal and stimulated an epithelial-like morphology with concomitant alterations in EMT markers expression, and reduced migration and invasion, thus causing the shift of the EMT balance in favor of an epithelial state. Our experiments provide new insights into the function of MARK2 in suppressing EMT and suggest that modulation of MARK2 expression is a potential approach to inhibit the invasiveness and migration of cervical cancer cells and the attendant pathologic processes including metastasis.

In summary, our results reveal that MARK2 plays a role in synergistically activating AMPK and reorganizing the actin cytoskeleton, and functions as an intracellular inhibitor for cell cycle progression and EMT in HeLa cells. The present study has provided a fascinating link between LKB1-mediated control of cell proliferation and cell polarity, which may benefit cancer research, but may also shed light on basic principles of epithelial biology.

\section{Acknowledgements}

The present study was supported by a grant from the National Natural Science Foundation of China (no. 81201544).

\section{References}

1. McGarrity TJ and Amos C: Peutz-Jeghers syndrome: Clinicopathology and molecular alterations. Cell Mol Life Sci 63: 2135-2144, 2006

2. Hearle N, Schumacher V, Menko FH, Olschwang S, Boardman LA, Gille JJ, Keller JJ, Westerman AM, Scott RJ, Lim W, et al: Frequency and spectrum of cancers in the Peutz-Jeghers syndrome. Clin Cancer Res 12: 3209-3215, 2006.

3. Ji H, Ramsey MR, Hayes DN, Fan C, McNamara K, Kozlowski P, Torrice C, Wu MC, Shimamura T, Perera SA, et al: LKB1 modulates lung cancer differentiation and metastasis. Nature 448: 807-810, 2007.

4. Lizcano JM, Göransson O, Toth R, Deak M, Morrice NA, Boudeau J, Hawley SA, Udd L, Mäkelä TP, Hardie DG, et al: LKB1 is a master kinase that activates 13 kinases of the AMPK subfamily, including MARK/PAR-1. EMBO J 23: 833-843, 2004.

5. Alessi DR, Sakamoto K and Bayascas JR: LKB1-dependent signaling pathways. Annu Rev Biochem 75: 137-163, 2006.

6. Ylikorkala A, Rossi DJ, Korsisaari N, Luukko K, Alitalo K, Henkemeyer $\mathbf{M}$ and Mäkelä TP: Vascular abnormalities and deregulation of $V E G F$ in $L k b 1$-deficient mice. Science 293: 1323$1326,2001$.

7. Jishage K, Nezu J, Kawase Y, Iwata T, Watanabe M, Miyoshi A, Ose A, Habu K, Kake T, Kamada N, et al: Role of Lkb1, the causative gene of Peutz-Jegher's syndrome, in embryogenesis and polyposis. Proc Natl Acad Sci USA 99: 8903-8908, 2002.

8. Williams T and Brenman JE: LKB1 and AMPK in cell polarity and division. Trends Cell Biol 18: 193-198, 2008.

9. Hardie DG: AMPK - sensing energy while talking to other signaling pathways. Cell Metab 20: 939-952, 2014.

10. Sebbagh M, Olschwang S, Santoni MJ and Borg JP: The LKB1 complex-AMPK pathway: The tree that hides the forest. Fam Cancer 10: 415-424, 2011.

11. Dasgupta B and Chhipa RR: Evolving lessons on the complex role of AMPK in normal physiology and cancer. Trends Pharmacol Sci 37: 192-206, 2016.

12. Fogarty S, Ross FA, Vara Ciruelos D, Gray A, Gowans GJ and Hardie DG: AMPK causes cell cycle arrest in LKB1-deficient cells via activation of CAMKK2. Mol Cancer Res 14: 683-695, 2016.

13. Chang HW, Lee YS, Nam HY, Han MW, Kim HJ, Moon SY, Jeon H, Park JJ, Carey TE, Chang SE, et al: Knockdown of $\beta$-catenin controls both apoptotic and autophagic cell death through LKB1/AMPK signaling in head and neck squamous cell carcinoma cell lines. Cell Signal 25: 839-847, 2013.

14. Liang $X$, Wang $P$, Gao $Q$ and Tao $X$ : Exogenous activation of LKB1/AMPK signaling induces $\mathrm{G}_{1}$ arrest in cells with endogenous LKB1 expression. Mol Med Rep 9: 1019-1024, 2014.

15. Okada T, Sinha S, Esposito I, Schiavon G, López-Lago MA, Su W, Pratilas CA, Abele C, Hernandez JM, Ohara M, et al: The Rho GTPase Rnd1 suppresses mammary tumorigenesis and EMT by restraining Ras-MAPK signalling. Nat Cell Biol 17: 81-94, 2015.

16. Chen N, Balasenthil S, Reuther J and Killary AM: DEAR1, a novel tumor suppressor that regulates cell polarity and epithelial plasticity. Cancer Res 74: 5683-5689, 2014.

17. Mohseni M, Sun J, Lau A, Curtis S, Goldsmith J, Fox VL, Wei C, Frazier M, Samson O, Wong KK, et al: A genetic screen identifies an LKB1-MARK signalling axis controlling the Hippo-YAP pathway. Nat Cell Biol 16: 108-117, 2014.

18. Shelly M and Poo MM: Role of LKB1-SAD/MARK pathway in neuronal polarization. Dev Neurobiol 71: 508-527, 2011.

19. Lee S, Wang JW, Yu W and Lu B: Phospho-dependent ubiquitination and degradation of PAR-1 regulates synaptic morphology and tau-mediated $\mathrm{A} \beta$ toxicity in Drosophila. Nat Commun 3: $1312,2012$.

20. Matenia D and Mandelkow EM: The tau of MARK: A polarized view of the cytoskeleton. Trends Biochem Sci 34: 332-342, 2009.

21. Müller J, Ritt DA, Copeland TD and Morrison DK: Functional analysis of C-TAK1 substrate binding and identification of PKP2 as a new C-TAK1 substrate. EMBO J 22: 4431-4442, 2003. 
22. Liang X, Xu G, Gao Q and Tao X: LKB1 expression reverses the tumorigenicity of L02 cells. Oncol Rep 36: 1055-1061, 2016.

23. Liang X, Wang P, Gao Q, Xiang T and Tao X: Endogenous LKB knockdown accelerates $\mathrm{G}_{1} / \mathrm{S}$ transition through $\mathrm{p} 53$ and $\mathrm{p} 16$ pathways. Cancer Biol Ther 9: 156-160, 2010.

24. Ladhani O, Sánchez-Martinez C, Orgaz JL, Jimenez B and Volpert OV: Pigment epithelium-derived factor blocks tumor extravasation by suppressing amoeboid morphology and mesenchymal proteolysis. Neoplasia 13: 633-642, 2011.

25. Tiainen M, Vaahtomeri K, Ylikorkala A and Mäkelä TP: Growth arrest by the LKB1 tumor suppressor: Induction of $\mathrm{p} 21^{\mathrm{WAF} 1 / \mathrm{CIP} 1}$ Hum Mol Genet 11: 1497-1504, 2002.

26. Fogarty S and Hardie DG: C-terminal phosphorylation of LKB1 is not required for regulation of AMP-activated protein kinase, BRSK1, BRSK2, or cell cycle arrest. J Biol Chem 284: 77-84, 2009.

27. Gopinath P and Ghosh SS: Apoptotic induction with bifunctional E.coli cytosine deaminase-uracil phosphoribosyltransferase mediated suicide gene therapy is synergized by curcumin treatment in vitro. Mol Biotechnol 39: 39-48, 2008.

28. Lee CJ, Jang JH, Lee JY, Lee MH, Li Y, Ryu HW, Choi KI, Dong Z, Lee HS, Oh SR, et al: Aschantin targeting on the kinase domain of mammalian target of rapamycin suppresses epidermal growth factor-induced neoplastic cell transformation. Carcinogenesis 36: 1223-1234, 2015.

29. Sclafani RA and Holzen TM: Cell cycle regulation of DNA replication. Annu Rev Genet 41: 237-280, 2007.

30. Tiainen M, Ylikorkala A and Mäkelä TP: Growth suppression by $L k b 1$ is mediated by a $G_{1}$ cell cycle arrest. Proc Natl Acad Sci USA 96: 9248-9251, 1999.

31. Gurumurthy S, Hezel AF, Sahin E, Berger JH, Bosenberg MW and Bardeesy N: LKB1 deficiency sensitizes mice to carcinogen-induced tumorigenesis. Cancer Res 68: 55-63, 2008.
32. Cánepa ET, Scassa ME, Ceruti JM, Marazita MC, Carcagno AL, Sirkin PF and Ogara MF: INK4 proteins, a family of mammalian CDK inhibitors with novel biological functions. IUBMB Life 59: 419-426, 2007.

33. Manning AL and Dyson NJ: pRB, a tumor suppressor with a stabilizing presence. Trends Cell Biol 21: 433-441, 2011.

34. Abbas T and Dutta A: p21 in cancer: Intricate networks and multiple activities. Nat Rev Cancer 9: 400-414, 2009.

35. Pei XH and Xiong Y: Biochemical and cellular mechanisms of mammalian CDK inhibitors: A few unresolved issues. Oncogene 24: 2787-2795, 2005.

36. Pei XH and Xiong Y: Biochemical and cellular mechanisms of mammalian CDK inhibitors: A few unresolved issues. Oncogene 24: 2787-2795, 2005.

37. Oei AL, van Leeuwen CM, ten Cate R, Rodermond HM, Buist MR, Stalpers LJ, Crezee J, Kok HP, Medema JP and Franken NA: Hyperthermia selectively targets human papillomavirus in cervical tumors via p53-dependent apoptosis. Cancer Res 75: 5120-5129, 2015.

38. Setogawa T, Shinozaki-Yabana S, Masuda T, Matsuura $\mathrm{K}$ and Akiyama T: The tumor suppressor LKB1 induces p21 expression in collaboration with LMO4, GATA-6, and Ldb1. Biochem Biophys Res Commun 343: 1186-1190, 2006.

39. Aktories K: Bacterial protein toxins that modify host regulatory GTPases. Nat Rev Microbiol 9: 487-498, 2011.

40. Mokady D and Meiri D: RhoGTPases - A novel link between cytoskeleton organization and cisplatin resistance. Drug Resist Updat 19: 22-32, 2015

41. Savagner P: The epithelial-mesenchymal transition (EMT) phenomenon. Ann Oncol 21 (Suppl 7): vii89-vii92, 2010.

42. Gandalovičová A, Vomastek T, Rosel D and Brábek J: Cell polarity signaling in the plasticity of cancer cell invasiveness. Oncotarget 7: 25022-25049, 2016. 